\title{
Highlights of 2008 in bone marrow biopsy pathology
}

\author{
Jon van der Walt
}

Received: 4 February 2009 / Accepted: 6 February 2009/Published online: 24 February 2009

(C) Springer-Verlag 2009

Advances in bone marrow pathology continued apace in 2008. Some fields saw increasing consensus, others vigorous debate! The following are a personal selection of the highlights.

\section{Myeloproliferative neoplasms}

The fiftieth anniversary of Blood saw the publication of a series of excellent review articles. The myeloproliferative disorders [1] were first characterised by Gustav Heuck who described myelofibrosis (MF) in 1879. Louis Henri Vaquez described polycythemia vera (PV) in 1892. Essential thrombocythemia (ET) followed somewhat later in 1934 when Emil Epstein and Alfred Goedel recognised it as a distinct clinical syndrome. William Dameshek, however, noted the overlapping clinical features of the three disorders and preferred to consider them 'closely interrelated'. This clinical observation has considerable relevance to later developments, in particular the identification of JAK2 ${ }^{\mathrm{V} 617 \mathrm{~F}}$ in all three myeloproliferative neoplasms (MPNs). Currently, $\mathrm{JAK} 2^{\mathrm{V} 617 \mathrm{~F}}$ gene dosage is thought to distinguish PV from $\mathrm{JAK} 2^{\mathrm{V} 617 \mathrm{~F}}$-positive ET. Familial studies have given support to the idea that additional inherited alleles are implicated in the pathogenesis of MPNs and that these remain unidentified. It is also possible that there are inherited modifiers that, whilst not predisposing to the development of MPN, may

J. van der Walt $(\bowtie)$

Department of Histopathology, St Thomas' Hospital,

Westminster Bridge Road,

London SE1 7EH, UK

e-mail: jon.vanderwalt@kcl.ac.uk contribute to the clinical phenotype of JAK2 ${ }^{\mathrm{V} 617 \mathrm{~F}}$-positive MPN. In addition, data suggest that there may be 'pre-JAK2' alleles responsible for clonal haemopoiesis that does not manifest as a clinical MPN. The progression of JAK2 ${ }^{\mathrm{V} 617 \mathrm{~F}}$ positive MPN to JAK2 ${ }^{\mathrm{V} 617 \mathrm{~F}}$-negative acute leukaemia supports the existence of a JAK2 $2^{\mathrm{V} 617 \mathrm{~F}}$-negative progenitor. Studies of JAK2 ${ }^{\mathrm{V} 617 \mathrm{~F}}$-negative MPNs have identified variant MPLW515L in JAK2 ${ }^{\mathrm{V} 617 \mathrm{~F}}$-negative ET associated with marked thrombocytosis and myelofibrosis.

A second excellent review on the history of myeloproliferative disorders before and after Dameshek gives further insight into the history of the haematology of the MPNs [2].

The revised 2008 World Health Organisation (WHO) classification included significant revisions to the classification of MPN, including their designation as 'neoplasms' and the introduction of 'primary myelofibrosis' as a replacement term for 'chronic idiopathic myelofibrosis' [3]. Clinical guidance and a useful series of diagnostic algorithms were published by Tefferi and Vardiman [4]. The importance of both the bone marrow biopsy and molecular characterisation is emphasised. The authors remind us that not all cases can be classified and these should be placed into the 'MPN, unclassifiable' category when this is the case and closely followed to monitor any changes that might warrant revision of the diagnosis.

Spivak and Silver [5] emphasised the variability of the phenotype of the MPNs and the changes that may take place over time as the disease progresses. The ongoing controversy over the reliability of histology of the bone marrow in distinguishing the categories of MPN and the ability to recognise a 'pre-fibrotic' phase of primary myelofibrosis is discussed and an alternative proposal based on clinical parameters is proposed. The biologically unsatisfactory adoption of 'primary' myelofibrosis was noted. It is clear that 
although substantial progress in molecular classification of PV, ET and MF has been made, there is much still to be done before unanimity exists in the classification of these disorders. It seems Dameshek was right. They are indeed 'closely interrelated'. Cytogenetic studies of BCR-ABL1-negative MPNs lend further support to this view. In a comprehensive review, Reilly [6] recorded that the incidence of cytogenetic abnormalities in MPNs varied from 30\% in $\mathrm{MF}$ to less than $5 \%$ in ET and that no specific abnormalities were found. These abnormalities may, however, be secondary subclones resulting from genetic instability of the original clone. Certain abnormalities such as trisomy 8 and $12 \mathrm{q}$ - may, however, indicate that an inferior prognosis and the cytogenetic profiling of MPNs is therefore of value [6].

The presence of JAK2 $2^{\mathrm{V} 617 \mathrm{~F}}$ may be of use in the distinction of fibrotic myelodysplastic syndrome (MDS) from primary myelofibrosis. Olsen et al. [7] analysed archived fixed material using real-time polymerase chain reaction (PCR) for $\mathrm{JAK} 2^{\mathrm{V} 617 \mathrm{~F}}$ and found that they could separate fibrotic MPN from myelodysplasia with fibrosis. PCR for JAK $2^{\mathrm{V} 617 \mathrm{~F}}$ in paraffin-embedded tissues may prove to be very useful in cases with morphology intermediate between MPN and fibrotic disorders.

\section{Myelodysplastic syndromes}

The International Working Group on Morphology of Myelodysplastic Syndrome published consensus proposals for the definition and enumeration of myeloblasts and ring sideroblasts [8]. The group reviewed the morphological features of bone marrows from all subtypes of myelodysplastic syndrome and agreed on a set of recommendations: (1) agranular or granular blast cells be defined (replacing the previous types I, II and III blasts), (2) dysplastic promyelocytes be distinguished from cytologically normal promyelocytes and from granular blast cells, (3) sufficient cells be counted to give a precise blast percentage, particularly at thresholds that are important for diagnosis or prognosis and (4) ring sideroblasts be defined as erythroblasts in which there are a minimum of five siderotic granules covering at least a third of the nuclear circumference. Clearer definitions are likely to improve precision in the diagnosis and classification of myelodysplastic syndrome in the context of the WHO classification. However, no immunocytochemical data were provided and the designation of 'blast' in the context of the bone marrow biopsy was not discussed. Caution is therefore needed in comparing counts of $\mathrm{CD} 34^{+}$progenitors in the bone marrow biopsy with the aspirate blast count.

The 5q- syndrome was first described in 1974 by Van den Berghe et al., and the condition has been recently reviewed [9]. In comparison with other types of MDS, 5q- syndrome is characterised by isolated $\operatorname{del}(5 \mathrm{q})$ and characteristic megakaryocytes with hypolobated nuclei. Of great interest is that lenalidomide therapy induces haematological remission which has been documented to occur in the absence of cytogenetic remission, suggesting that the action of lenalidomide is not specifically on the $5 \mathrm{q}$ - clone. It is suggested that loss of an RPS14 allele accounts for the characteristic phenotype, but further investigation is required.

\section{The myelodysplastic/myeloproliferative neoplasms}

Historical difficulties in classifying disease with dysplastic marrows that also showed proliferation lead to the WHO classification introducing the MDS/MPN category to provide a less restrictive definition [10]. This 'spotlight review' [10] provides a concise overview of the MDS/MPN category which includes atypical chronic myeloid leukaemia (aCML), chronic myelomonocytic leukaemia (CMML), MDS/MPN, unclassifiable (MDS/MPNu), refractory anaemia with ring sideroblasts and thrombocytosis (RARS-T), 5q-/JAK2-positive atypical myeloproliferative disease and atypical myeloproliferative disease associated with isolated isochromosome 17q. It is emphasised that morphological features are often not diagnostic and that a multiparameter approach is required for the 'clinicopathological assignation' of these entities. By definition, the Philadelphia chromosome or $B C R / A B L 1$ fusion gene is absent, and in fact, no specific genetic defects have been identified. Likewise, cases with rearranged PDGFRA or PDGFRB genes are now classified separately. Attention to both peripheral blood and bone marrow is required, exemplified by the distinction of aCML from typical CML by the presence of peripheral dysgranulopoiesis and lack of basophilia. Whilst the classical manifestation of CMML is peripheral monocytosis, rare cases fall below the $1000 \times$ $10 \% / \mathrm{L}$ threshold and the presence of bone marrow monocyte proliferation allows the diagnosis of 'bone marrow predominant' CMML. Immunocytochemistry is of importance in the diagnosis [10] and may show architectural features such as 'myelomonocytic' nodules not apparent in the aspirate [11].

Juvenile myelomonocytic leukaemia (JMML) presents between birth and 6 years of age [12] and lacks any causal chromosomal abnormalities. However, the pathogenesis is known to involve dysregulation and aberrant signal transduction of the Ras pathway, providing a model for the investigation of these abnormalities and through its association with neurofibromatosis type 1 and Noonan syndrome, a teaching tool for the exploration of inherited predispositions to cancer [12]. 


\section{Myeloid and lymphoid neoplasms with eosinophilia and abnormalities of PDGFRA, PDGFRB and FGFR1}

Five years have passed since the identification of FIP1L1-PDGFRA fusion gene [13], and the importance of this finding has been recognised in the revised WHO classification [3]. In a comprehensive review, Gotlib and Cools describe the molecular separation of these clonal eosinophilias from reactive eosinophilia associated with infection, hypersensitivity, lymphomas, other myeloid neoplasms and $\mathrm{T}$ cell populations with aberrant phenotype and abnormal cytokine production. Several variant PDGFRA, PDGFRB and FGFRI fusion genes have been identified often in unique case reports. PDGFRB rearrangements are rare in other defined entities such as JMML, aCML, etc., but recognition is essential in view of the sensitivity of such cases to imatinib [13]. Systemic mastocytosis with eosinophilia has also been shown to be FIP1L1-PDGFRA-positive. The dramatic response to imatinib is highlighted.

\section{Lymphoid lesions in the bone marrow}

Sézary syndrome (SS) is often considered to represent a leukaemic form of mycosis fungoides (MF). However, the 2008 edition of the WHO classification lists SS and MF as separate lymphomas. Van Doorn et al. [14] provide support for this view. Using array-based comparative genomic hybridisation and microarray analysis of gene expression, chromosomal alterations in MF were characterised by gains on chromosomes 1 and 7 and loss on 9, whereas SS demonstrated gains on chromosomes 8 and 17 and loss on 10. These changes suggest that the pathogenesis of MF and SS follow different pathways and are distinct.

Splenic marginal zone lymphoma with circulating villous lymphocytes (SMZL) generally has an indolent clinical course, but is known to progress. Dungarwalla et al. [15] studied 48 cases of SMZL and found progression in 31. The cases that progressed to large B cell lymphoma had significantly greater peripheral lymph node involvement and a higher incidence of serum IgM paraprotein. The rate of transformation to large B cell lymphoma was 19\% (9/ 48). In four cases, the transformation was in the bone marrow. The prognosis was worse in these cases compared with those with lymph node transformation, being refractory to treatment. This paper emphasises the importance of a bone marrow biopsy in patients with SMZL showing evidence of clinical progression.
Conflict of interest The author declares no conflict of interest.

\section{References}

1. Levine RL, Gilliland DG (2008) Myeloproliferative disorders. Blood 112:2190-2198

2. Tefferi A (2008) The history of myeloproliferative disorders: before and after Dameshek. Leukemia 22:3-13

3. Swerdlow SH, Campo E, Harris NL, Jaffe ES, Pileri SA, Stein H, Thiele J, Vardiman JW (eds) (2008) WHO classification of tumours of the haematopoietic and lymphoid tissues. IARC, Lyon

4. Tefferi A, Vardiman JW (2008) Classification and diagnosis of myeloproliferative neoplasms: the 2008 World Health Organization criteria and point-of-care diagnostic algorithms. Leukemia 22:14-22

5. Spivak JL, Silver RT (2008) The revised World Health Organization diagnostic criteria for polycythemia vera, essential thrombocythemia and primary myelofibrosis: an alternative proposal. Blood 112:231-239

6. Reilly (2008) Pathogenetic insight and prognostic information from standard and molecular cytogenetic studies in the BCRABL-negative myeloproliferative neoplasms (MPNs). Leukemia 22:1818-1827

7. Olsen RJ, Dunphy CH, O'Malley DP, Rice L, Ewton AA, Chang $\mathrm{CC}$ (2008) The implication of identifying JAK $2^{\mathrm{V} 617 \mathrm{~F}}$ in myeloproliferative neoplasms and myelodysplastic syndrome with bone marrow fibrosis. J Hematopathol 1:111-117

8. Mufti GJ, Bennett JM, Goasguen J, Bain BJ, Baumann I, Brunning R, Cazzola M, Fenaux P, Germing U, HellströmLindberg E, Jinnai I, Manabe A, Matsuda A, Niemeyer CM, Sanz G, Tomonaga M, Vallespi T, Yoshimi A, International Working Group on Morphology of Myelodysplastic Syndrome (2008) Diagnosis and classification of myelodysplastic syndrome: International Working Group on Morphology of myelodysplastic syndrome (IWGM-MDS) consensus proposals for the definition and enumeration of myeloblasts and ring sideroblasts. Haematologica 93:1712-7

9. Mohamedali A, Mufti GJ (2008) Van den Berghe' 5q- syndrome in 2008. B J Haematol 144:157-168

10. Orazi A, Germing U (2008) The myelodysplastic/myeloproliferative neoplasms: myeloproliferative neoplasms with dysplastic features. Leukemia 22:1308-1319

11. Ngo NT, Lampert IA, Naresh KN (2008) Bone marrow trephine morphology and immunohistochemical findings in chronic myelomonocytic leukaemia. B J Haematol 141:771-781

12. Emanuel PD (2008) Juvenile myelomonocytic leukaemia and chronic myelomonocytic leukaemia. Leukemia 22:1335-1342

13. Gotlib J, Cools J (2008) Five years since the discovery of FIP1L1PDGFRA; what we have learned about the fusion and other molecularly defined eosinophilias. Leukemia 22:1999-2010

14. Van Doorn R, van Kester MS, Dijkman R, Vermeer MH, Mulder AA, Szuhai K, Knijnenburg J, Boer JM, Willemze R, Tensen CP (2009) Oncogenomic analysis of mycosis fungoides reveals major differences with Sézary syndrome. Blood 113:127-136

15. Dungarwalla M, Appiah-Cubi S, Kulkarni S, Saso R, Wotherspoon A, Osuji N, Swansbury J, Cunningham DC, Catovsky D, Dearden CE, Matutes E (2008) High grade transformation in splenic marginal zone lymphoma with circulating villous lymphocytes: site of transformation influences response to therapy and prognosis. B J Haematol 143:71-74 